Instituto Internacional de Investigación y Desarrollo Tecnológico Educativo INDTEC, C.A.

DOI: https://doi.org/10.29394/Scientific.issn.2542-2987.2020.5.16.12.230-245

OAI-PMH: http://www.indteca.com/ojs/index.php/Revista Scientific/oai

Artículo Original / Original Article

\title{
Motivación hacia la Práctica de la Actividad Físico-Deportiva y Recreativas de los Estudiantes Secundarios
}

\author{
Autor: Olger Estuardo Almache Tello \\ Universidad Nacional de Educación, UNAE \\ estuardo.almache@unae.edu.ec \\ Azogues, Ecuador \\ https://orcid.org/0000-0003-4620-9646
}

\section{Resumen}

El objetivo de este trabajo investigativo es el detectar la orientación motivacional de los estudiantes secundarios de las unidades educativas de la ciudad de Azogues a la práctica de la actividad física y recreativa, de igual manera el grado de noción que tienen el alumnado sobre la oferta deportiva de su institución educativa, este trabajo investigativo se realizó sobre una muestra de 308 (197 hombres y 111 Mujeres) estudiantes de las unidades educativas La Salle, Luis Rogerio González y Unidad Universitaria de Azogues, todas ellas pertenecientes a la ciudad de Azogues, provincia del Cañar, con edades comprendidas entre 16 y 18 años el muestreo que se realizo fue de carácter aleatorio, para la valoración de las variables se efectuó un estudio social de carácter cuantitativo para lo cual se validó y diseño dos cuestionarios con las siguiente dimensiones: uno sobre hábitos de práctica físico-deportiva y recreativa y otro para evidenciar si los encuestados conocían los servicios deportivos que presta su institución educativa. Los resultados reflejan que El $71.9 \%$ del estudiantado investigado expresan que no tienen información, sobre las actividades recreativas y deportivas que se desarrolla en su institución educativa, mientras que el $16 \%$ indica que realiza actividad física por recreación, y un $44 \%$ de estudiantes exteriorizan que realizan actividad física por salud estas son las razones principales que estimulan a los estudiantes secundario de la ciudad de Azogues a realizar actividad física.

Palabras clave: motivación; alumno; orientación.

Cómo citar este artículo:

Almache, O. (2020). Motivación hacia la Práctica de la Actividad Físico-Deportiva y Recreativas de los Estudiantes Secundarios. Revista Scientific, 5(16), 230-245, e-ISSN: 2542-2987. Recuperado de: https://doi.org/10.29394/Scientific.issn.2542-2987.2020.5.16.12.230-245

Fecha de Recepción: 09-12-2019
Fecha de Aceptación:

30-03-2020
Fecha de Publicación: 05-05-2020 
OAl-PMH: http://www.indteca.com/ojs/index.php/Revista_Scientific/oai

Artículo Original / Original Article

\title{
Motivation towards the Practice of Physical-Sports and Recreational Activity of Secondary Students
}

\begin{abstract}
The objective of this research work is to detect the motivational orientation of the secondary students of the educational units of the city of Azogues to the practice of physical and recreational activity, in the same way the degree of notion that the students have about the sports offer from their educational institution, this research work was carried out on a sample of 308 (197 men and 111 women) students from the La Salle educational units, Luis Rogerio González and the University Unit of Azogues, all of them belonging to the city of Azogues, province of Cañar, with ages between 16 and 18 years, the sampling that was carried out was random, for the evaluation of the variables a quantitative social study was carried out, for which two questionnaires with the following dimensions were validated and designed: one on physical-sports and recreational practice habits and another to show if the respondents knew sports services provided by your educational institution. The results reflect that $71.9 \%$ of the investigated students express that they do not have information on the recreational and sports activities that take place in their educational institution, while $16 \%$ indicate that they carry out physical activity for recreation, and $44 \%$ of students externalize that carry out physical activity for health these are the main reasons that stimulate the secondary students of the city of Azogues to carry out physical activity.
\end{abstract}

Keywords: motivation; student; counselling.

How to cite this article:

Almache, O. (2020). Motivation towards the Practice of Physical-Sports and Recreational Activity of Secondary Students. Revista Scientific, 5(16), 230-245, e-ISSN: 2542-2987. Recovered from: https://doi.org/10.29394/Scientific.issn.2542-2987.2020.5.16.12.230-245

Date Received:

09-12-2019
Date Acceptance:

30-03-2020
Date Publication: 05-05-2020 


\section{Introducción}

Este trabajo es producto de un análisis minucioso partiendo de la premisa de la deserción del deporte de estudiantes que pasan de la secundaria a la vida universitaria, trayendo consigo un sinnúmero de problemas en lo social y de salud, diversos estudios realizados sobre La actividad físicodeportiva y recreativa entre los más importantes

De acuerdo con Devis (2000): se destaca la necesidad de conocer todas las formas de integrar al individuo a la participación individual y organizada dentro de la actividad física buscando un equilibrio entre la práctica y el buen uso del tiempo libre, de igual forma se ha convertido en uno de los movimientos con mayor trascendencia en la sociedad el incremento de personas que realizan deporte, y la visión que el deporte es salud ha permitido en la sociedad actual la construcción de una cultura deportiva que motiva a la sociedad a participar cada vez más en diversas actividades físicas, cabe indicar que la mayoría de personas realizan actividades físicas con el único fin de recrearse, mientras que otras lo hacen para medir sus condiciones físicas y obtener un logro en una competencia.

Algunos estudios, como el de Álvarez (2010), manifiesta que: "la falta de actividad física es un riesgo para la salud mundial y es un problema extendido y en rápido ascenso en los países, tanto desarrollados como en los que están en vías de desarrollo" (pág. 55); de este modo para lograr resultados significativos en la prevención de las enfermedades, es necesario que los medios y las políticas públicas que opten cada estado reconozcan el papel fundamental que juega la actividad física en nuestras sociedades y en especial en nuestros jóvenes.

Según Ferrando (2006), se manifiesta:

La necesidad de analizar el deporte como algo más que una simple distracción, lleva a un estudio como elemento integrador en la vida de los individuos y de los grupos sociales, que ejercen una influencia cada vez mayor sobre ellos (pág. 27). 
En la sociedad actual, el deporte ha pasado a ser un fenómeno social muy importante pues establece la interrelación del hombre con pautas sociales y culturales, de tal forma que llega a ser muy complejo establecer cuáles son los límites de la conducta propiamente deportiva.

En los establecimientos educativos secundarios, la práctica de la actividad física deportiva y recreativa, debe anclarse a la situación sociocultural y económica, la misma que permitirá implementar proyectos deportivos acordes a una realidad situacional.

A su vez, Fernández (2010): mantiene la necesidad de analizar, los motivos por los cuales la población practica actividad física recreativa o permanecen en la inactividad, para diseñar estrategias que permita a la población estudiantil incrementar el tiempo de práctica de la Actividad física con el objetivo de promover la salud y bienestar.

Por consiguiente, Moreno, Martínez y Torres (2012): resaltan la "importancia de conocer el tipo de motivación que hace que el número de personas que practican deportes o actividad física descienda de forma vertiginosa" (pág. 12); en conclusión, es imperiosa la necesidad de realizar acciones de concientización, para todos los fragmentos de la sociedad sobre los beneficios que trae la práctica deportiva.

Paralelamente, Balderrama-Ruedas, Díaz-Domínguez y GómezCastillo (2015), resaltan que la:

Falta de cultura hacia estas actividades han llevado a tener una población muy sedentaria y con resultados no muy favorables en algunos ámbitos como el deportivo, académico y un gran número de problemas de salud en comparación con otros países (pág. 223).

Es necesario recalcar que el objetivo fundamental de este trabajo investigativo pretende identificar las razones por lo que los alumnos de las unidades educativas de la ciudad de Azogues, realizan o no actividad física 
recreativa en sus unidades educativas, de igual manera, trata de saber si conocen la oferta deportiva que tienen las instituciones a las que pertenecen en el campo de la actividad física, con esta información levantada pretendemos mejorar el fomento y desarrollo de la actividad físico-deportiva y recreativa en el sistema educativo secundario de la ciudad.

Tomando en consideración que las instituciones educativas son autónomas en la parte administrativa y lo pueden normar acciones en bien del fomento al deporte. En este mismo orden de ideas, Riffo (2019): destaca que la administración es un proceso que se norme y se regula de acuerdo con las circunstancias, que, aunque existan estamentos legales predeterminados para el quehacer educativo, no siempre se podrá dar el mismo tratamiento a todas las situaciones que imperen, esto dependerá de lo interno y externo que fluctúe en cada institución educativa.

\section{Materiales y métodos}

Tabla 1. Ficha para la selección de la muestra.

\begin{tabular}{|c|l|}
\hline Ámbito & $\begin{array}{l}\text { Estudiantes de los Terceros del Bachillerato General } \\
\text { Unificado de las Unidades educativas: La Salle, Luis } \\
\text { Rogerio González y Unidad Educativa Particular } \\
\text { Universitaria de Azogues. }\end{array}$ \\
\hline Tipo de muestreo & Aleatorio simple. \\
\hline $\begin{array}{c}\text { Tamaño de la } \\
\text { muestra }\end{array}$ & 308 estudiantes (111 mujeres y 197 hombres). \\
\hline Instrumentos & $\begin{array}{l}\text { Cuestionario hábitos de la práctica físico-deportiva y } \\
\text { recreativas. }\end{array}$ \\
\hline Temporalización & Enero - abril 2019. \\
\hline
\end{tabular}

Fuente: El Autor (2019).

Este artículo se realizó con un modelo de 308 alumnos (111 mujeres y 197 hombres) de los dos géneros de la Unidad Educativa Particular La Salle, Unidad Educativa Luis Rogerio González y la Unidad Educativa Particular Universitaria de Azogues, pertenecientes a la ciudad de Azogues, provincia 
del Cañar, todos los estudiantes procedieron a llenar los cuestionarios de forma anónima y voluntaria, los estudiantes cursan el Tercero de Bachillerato General Unificado y se encuentran matriculados en el periodo académico 2018-2019. En la tabla 1, se expone un resumen de lo que se utilizó para la selección de la muestra.

La media en la edad de los estudiantes participantes en la encuesta fue de 17 años. De igual manera en la tabla 2, se encuentran las Unidades Educativas elegidas y la distribución de los estudiantes por unidad educativa, esta investigación cumple con los principios sobre confidencialidad, de igual manera a todos los encuestados se les pidió su consentimiento y se les explico claramente los objetivos de la investigación.

Tabla 2. Distribución de la muestra de estudiantes por Unidad Educativa.

\begin{tabular}{|l|c|c|}
\hline Unidad Educativa La Salle & Paralelo & Muestra \\
\hline Tercero "BGU" & "A" & 31 \\
\hline Tercero "BGU" & "B" & 31 \\
\hline Tercero "BGU" & "C" & 31 \\
\hline Unidad Educativa Luis Rogerio González & Paralelo & Muestra \\
\hline Tercero "BGU" Mecánica & "A" & 26 \\
\hline Tercero "BGU" Mecánica & "B" & 25 \\
\hline Tercero "BGU" Electricidad & "A" & 26 \\
\hline Tercero "BGU" Electricidad & "B" & 25 \\
\hline Tercero "BGU" Comercio & "A" & 26 \\
\hline Tercero "BGU" Comercio & "B" & 26 \\
\hline Unidad Educativa Particular Universitaria de Azogues & Paralelo & Muestra \\
\hline Tercero "BGU" & "A" & 31 \\
\hline Tercero "BGU" & "B" & 30 \\
\hline \multicolumn{1}{|c|}{ TOTAL } & & 308 \\
\hline
\end{tabular}

Fuente: El Autor (2019).

Los principios de inclusión fueron: participar libremente en la investigación, estar matriculado durante el periodo escolar 2018-2019 en el tercero del Bachillerato General Unificado, no realizar deporte o actividad física y recreativa. Los criterios de exclusión fueron: presentar alguna discapacidad física o psíquica que le impidiese responder el cuestionario. 


\subsection{Cuestionario de hábitos de práctica físico-deportiva y recreativa}

El instrumento de investigación fue elaborado con el propósito de levantar información: 1). Información sobre los participantes en la encuesta; 2). Conocimiento y valoración de las actividades deportivas ofertadas por su Unidad Educativa; y 3). Información sobre la práctica de actividades físicodeportivas y recreativas.

Par levantar información se utilizó la encuesta como técnica cuantitativa que consiste en una investigación realizada sobre una muestra de sujetos para fines estadísticos, con este propósito se realizó un cuestionario de preguntas sobre conductas hacia la actividad físico-deportiva y recreativa, mismas que guardan relación con el tema de investigación. En la tabla 3, se describe con más detalle los contenidos del cuestionario.

Tabla 3. Contenido del cuestionario sobre hábitos físico-deportivos y recreativos.

\begin{tabular}{|l|l|}
\hline \multicolumn{2}{|c|}{ COMPONENTES DEL CUESTIONARIO SOBRE HÁBITOS FÍSICO- } \\
DEPORTIVOS
\end{tabular}

Fuente: El Autor (2019).

\section{Resultados}

En relación con esta temática, en la tabla 4 y el gráfico 1, la mayoría de los estudiantes de las unidades educativas, manifiestan practicar actividad físico-deportiva "en grupo" (36.7\%), "solos" (35,8\%), un (22.8\%), lo realizan "en equipos deportivos" y el $(4,7 \%)$, en "actividades dirigidas de grupo". 
Instituto Internacional de Investigación y Desarrollo Tecnológico Educativo INDTEC, C.A.

DOI: https://doi.org/10.29394/Scientific.issn.2542-2987.2020.5.16.12.230-245

OAl-PMH: http://www.indteca.com/ojs/index.php/Revista_Scientific/oai

Artículo Original / Original Article

Tabla 4. Valoración: ¿Cómo practicas la actividad físico-deportiva y recreativa?

\begin{tabular}{|l|c|c|}
\hline \multicolumn{1}{|c|}{ Ítems } & Porcentaje & Cantidad \\
\hline En grupo & $36,7 \%$ & 93 \\
\hline Solo/Sola & $35,8 \%$ & 91 \\
\hline En equipos deportivos & $22.8 \%$ & 58 \\
\hline En actividad dirigidas de grupo & $4,7 \%$ & 12 \\
\hline Total & $100 \%$ & 254 \\
\hline
\end{tabular}

Fuente: El Autor (2019).

Gráfico 1. ¿Cómo practicas la actividad físico-deportiva y recreativa?

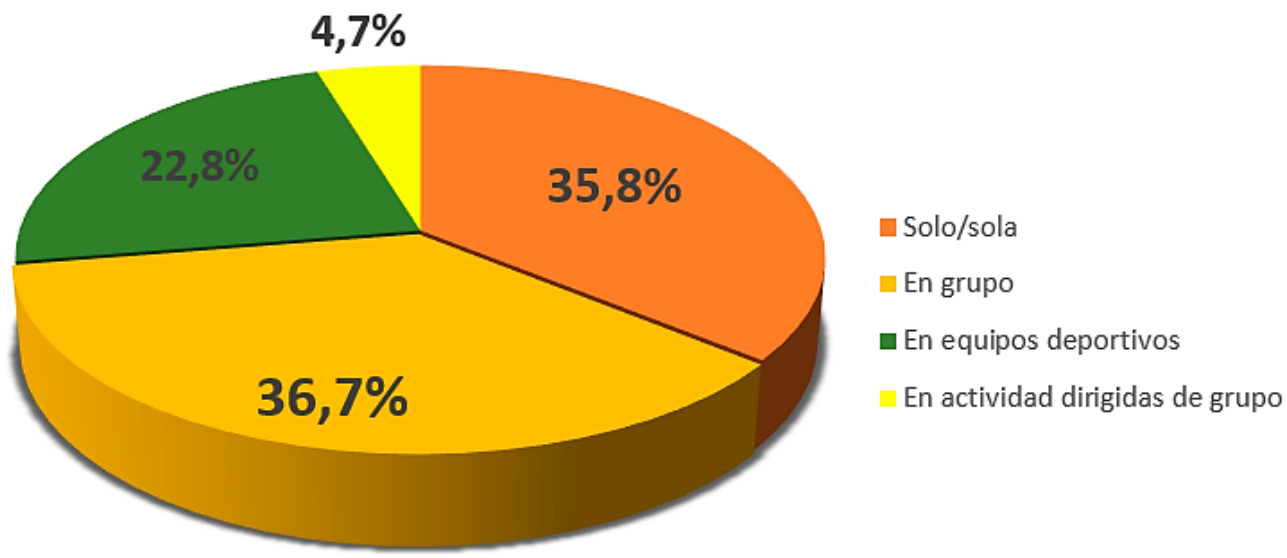

Fuente: El Autor (2019).

Las principales motivaciones para realizar actividades físico-deportivas y recreativa entre los estudiantes de las unidades educativas estudiadas aparecen bien determinadas: "salud" (43,3\%), "lúdico o juego" (16,5\%), "por estar con amigos" (14,2\%), "estética/verme bien" (12,3\%), "competir" (9,8\%), "otras" con un $(3,2 \%)$, y por obligación $(0,7 \%)$, estudios similares en el Ecuador, dan resultados que nos advierten una vital importancia por las vertientes "saludable" y "lúdico/recreativa" y que se desprende de la práctica físico-deportiva y recreativa, como se observa en la tabla 5 y el gráfico 2. 
Instituto Internacional de Investigación y Desarrollo Tecnológico Educativo INDTEC, C.A.

DOI: https://doi.org/10.29394/Scientific.issn.2542-2987.2020.5.16.12.230-245

OAI-PMH: http://www.indteca.com/ojs/index.php/Revista_Scientific/oai

Artículo Original / Original Article

Tabla 5. Valoración. ¿Cuál es la razón principal para realizar la actividad físicodeportiva y recreativa?

\begin{tabular}{|c|c|c|}
\hline Ítems & Porcentaje & Cantidad \\
\hline Salud & $43,3 \%$ & 110 \\
\hline Lúdico o juego & $16,5 \%$ & 42 \\
\hline Por estar con amigos & $14,2 \%$ & 36 \\
\hline Estética / verme bien & $12,3 \%$ & 31 \\
\hline Competir & $9,8 \%$ & 25 \\
\hline Otros & $3,2 \%$ & 8 \\
\hline Obligación & $0,7 \%$ & 2 \\
\hline Total & $100 \%$ & 254 \\
\hline
\end{tabular}

Fuente: El Autor (2019).

Gráfico 2. ¿Cuál es la razón principal para realizar la actividad físico-deportiva y recreativa?

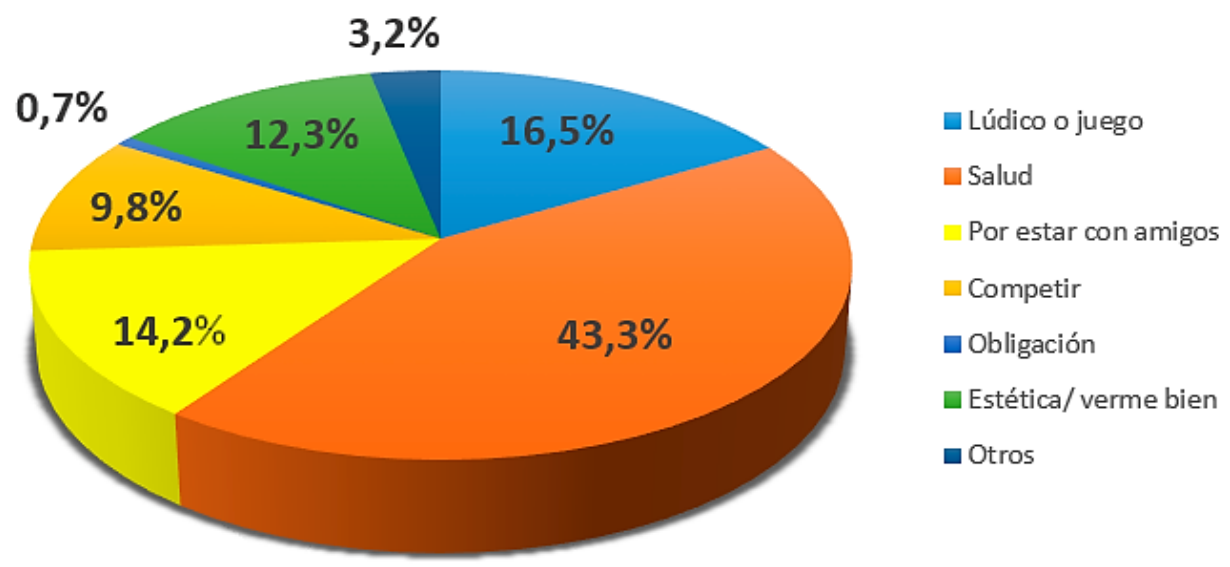

Fuente: El Autor (2019).

Los estudiantes de las unidades educativas estudiadas, vinculan la práctica físico-deportiva a: "los entretenimientos" (19,6\%), "la competencia" (16,6\%), "la diversión" (15,8\%), "permite adquirir una buena forma física" el $(14,2 \%)$, "mejora la salud" el (12,9\%), "permite estar con amigos" el (12,3\%), "sirve para relajarse" el $(6,2 \%)$, "es una forma de educación" $(1,2 \%)$, y "otros" $(1,2 \%)$, como se representa en la tabla 6 y gráfico 3 . Esta información no 
Instituto Internacional de Investigación y Desarrollo Tecnológico Educativo INDTEC, C.A.

DOI: https://doi.org/10.29394/Scientific.issn.2542-2987.2020.5.16.12.230-245

OAI-PMH: http://www.indteca.com/ojs/index.php/Revista_Scientific/oai

\section{Artículo Original / Original Article}

coincide con estudios realizado sobre el tema en la cual la mayoría de los encuestados asociaron el deporte a la salud.

Tabla 6. ¿Qué es lo que más te gusta de la práctica de la actividad físicodeportiva y recreativa?

\begin{tabular}{|l|c|c|}
\hline \multicolumn{1}{|c|}{ Ítems } & Porcentaje & Cantidad \\
\hline El entrenamiento & $19.6 \%$ & 50 \\
\hline La competición & $16,6 \%$ & 42 \\
\hline Por diversión & $15,8 \%$ & 40 \\
\hline Permite adquirir una buena forma física & $14,2 \%$ & 36 \\
\hline Mejora la salud & $12,9 \%$ & 33 \\
\hline Permite estar con amigos & $12,3 \%$ & 31 \\
\hline Sirve para relajarse & $6,2 \%$ & 16 \\
\hline Es una forma de educación & $1,2 \%$ & 3 \\
\hline Otros & $1,2 \%$ & 3 \\
\hline Total & $100 \%$ & 254 \\
\hline
\end{tabular}

Fuente: El Autor (2019).

Gráfico 3. ¿Qué es lo que más te gusta de la práctica de la actividad físicodeportiva y recreativa?

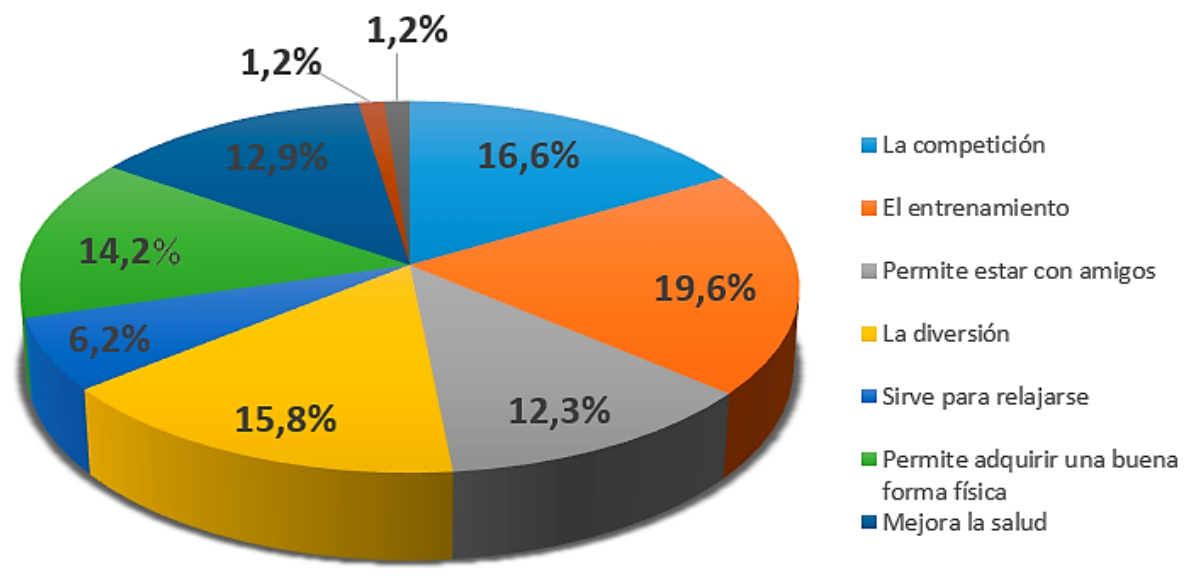

Fuente: El Autor (2019).

Es elevado el porcentaje de estudiantes de las unidades educativas que no utilizan las instalaciones deportivas de su institución, para realizar deporte 
o recreación en un $(38,4 \%)$, es un dato importante que el propio estudio considera como alarmante, un $(37,7 \%)$, lo realiza en las instalaciones de su institución y un $(23,9 \%)$ no utiliza por otra causa, resultados que debe llevar a la reflexión, la propia institución educativa, datos que se han plasmado en la tabla 7 y gráfico 4 .

Tabla 7. Valoración ¿Practicas actividad físico-deportiva y recreativa en las instalaciones deportivas de tu institución educativa?

\begin{tabular}{|l|c|c|}
\hline \multicolumn{1}{|c|}{ Ítems } & Porcentaje & Cantidad \\
\hline No & $38,4 \%$ & 93 \\
\hline $\mathrm{Si}$ & $37,7 \%$ & 91 \\
\hline Otras causas & $23,9 \%$ & 58 \\
\hline Total & $100 \%$ & 242 \\
\hline
\end{tabular}

Fuente: El Autor (2019).

Gráfico 4. ¿Practicas actividad físico-deportiva y recreativa en las instalaciones deportivas de tu institución educativa?

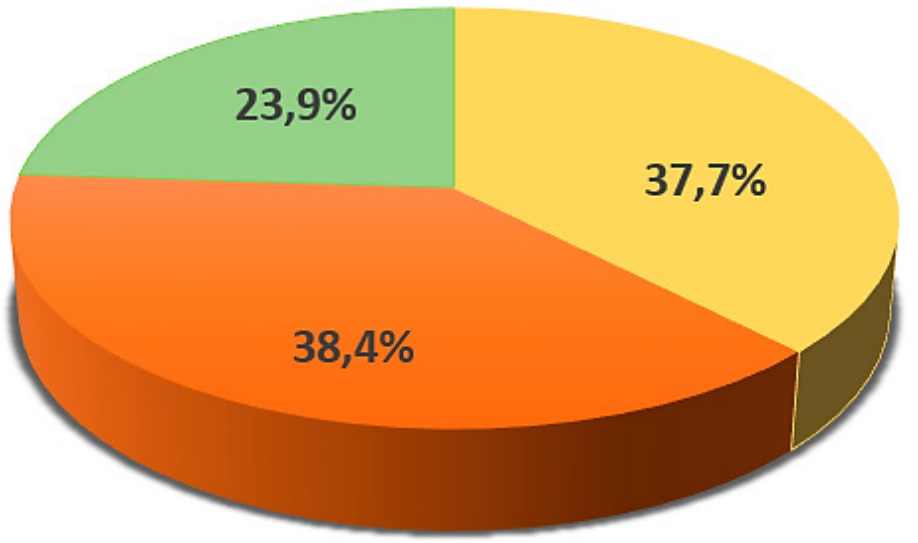

Fuente: El Autor (2019).

Un $(46,8 \%)$, de encuestados indican que la información que emite la institución sobre los servicios deportivos es "poca", un (35,4\%), nos indica que es suficiente la información, un $(11,2 \%)$, nos señalan que mucha y un $(6,6 \%)$, 
no tiene información, como se puede visualizar en la tabla 8 y gráfico 5 . Estos resultados son alarmantes por cuanto que en las instituciones educativas investigadas no cuentan con un proyectos, programas o estrategias que incentiven hacia la práctica deportiva de sus educandos.

Tabla 8. Valoración ¿Cuál es el grado de información sobre los servicios deportivos que presta su institución educativa?

\begin{tabular}{|l|c|c|}
\hline \multicolumn{1}{|c|}{ Ítems } & Porcentaje & Cantidad \\
\hline Poca & $46,8 \%$ & 119 \\
\hline Suficiente & $35,4 \%$ & 90 \\
\hline Mucha & $11,2 \%$ & 28 \\
\hline Nada & $6,6 \%$ & 17 \\
\hline Total & $100 \%$ & 254 \\
\hline
\end{tabular}

Fuente: El Autor (2019).

Gráfico 5. ¿Cuál es el grado de información sobre los servicios deportivos que presta su institución educativa?

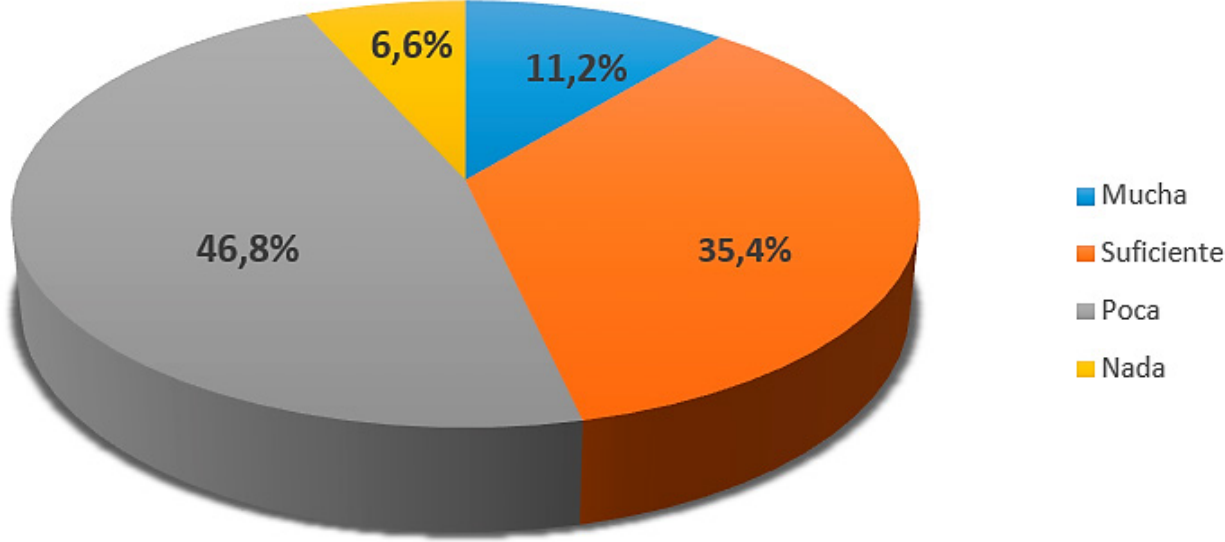

Fuente: El Autor (2019).

La razón principal por lo que los estudiantes encuestados explican la no participación en actividades deportivas y recreativas es la "falta de tiempo" $(35,1 \%)$, "falta de recursos económicos" un $(24,7 \%)$, por "enfermedad - lesión" un $(14,8 \%)$, "falta de oferta deportiva" un $(12,5 \%)$, "surgieron objetivos más 
importantes en mi vida" $(11,1 \%)$, y "falta de apoyo social" $(1,8 \%)$, como se exhibe en la tabla 9 y gráfico 6 . Este resultado es similar con otras investigaciones anteriores que también se encontraron con la "falta de tiempo", como una razón para no practicar actividad deportiva de manera regular.

Tabla 9. Valoración ¿Cuál es el motivo por el que no practicas actividad físicodeportiva y recreativa?

\begin{tabular}{|l|c|c|}
\hline \multicolumn{1}{|c|}{ Ítems } & Porcentaje & Cantidad \\
\hline Falta de tiempo & $35,1 \%$ & 19 \\
\hline Falta de recursos económicos & $24,7 \%$ & 13 \\
\hline Enfermedad - lesión & $14,8 \%$ & 8 \\
\hline Falta de oferta deportiva & $12,5 \%$ & 7 \\
\hline Surgieron objetivos más importantes en mi vida & $11,1 \%$ & 6 \\
\hline Falta de apoyo social & $1,8 \%$ & 1 \\
\hline Total & $100 \%$ & 54 \\
\hline
\end{tabular}

Fuente: El Autor (2019).

Gráfico 6. ¿Cuál es el motivo por el que no practicas actividad físico-deportiva y recreativa?
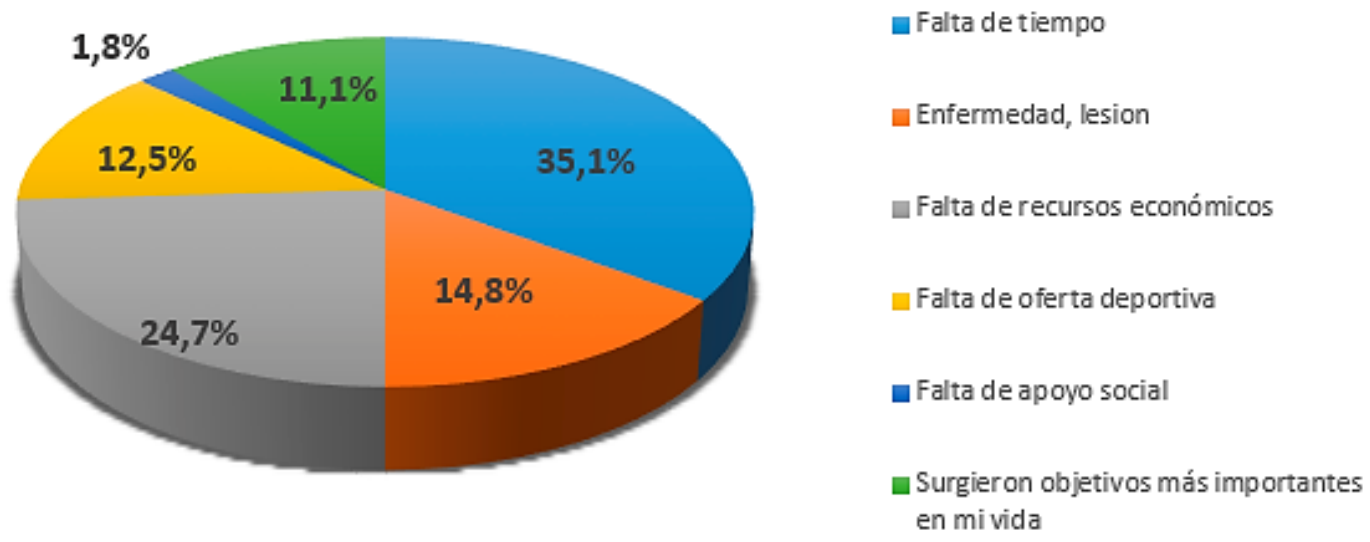

Fuente: El Autor (2019).

\section{Conclusiones}

No hay líneas objetivamente delimitadas en la forma seleccionada por 
lo estudiantes encuestados para la realización de la práctica físico-deportiva y recreativa, la práctica en equipos o solitario, exteriorizan porcentajes similares a la totalidad de la población.

Los estudiantes encuestados que practican actividad física tienen su inclinación por la actividad lúdica y saludable que la práctica físico-deportiva les provee. En efecto, los servicios enfocados a la mejora de la salud, la recreación y el entretenimiento a través de las actividades físico-deportivas son las que pueden tener mayor éxito entre los estudiantes.

La totalidad de los estudiantes encuestados que realizan la actividad físico-deportiva y recreativa, no usan las instalaciones deportivas de su institución educativa. La razón principal que plantean los estudiantes encuestados para no realizar la práctica físico-deportiva y recreativa, es "la falta de tiempo".

El estudio demuestra que existe un alto porcentaje de estudiantes encuestados, que indican que tienen poca información sobre la oferta de los servicios deportivos que presta la institución educativa a los estudiantes.

\section{Referencias}

Álvarez, F. (2010). Sedentarismo y actividad física. Revista Finlay, 10(Número especial), 55-60, e-ISSN: 2221-3434. Recuperado de:

http://www.revfinlay.sld.cu/index.php/finlay/article/view/10/10

Balderrama-Ruedas, J., Díaz-Domínguez, P., \& Gómez-Castillo, R. (2015).

Activación física y deporte: su influencia en el desempeño académico. Ra Ximhai, 11(4), 221-230, e-ISSN: 1665-0441. Recuperado de: https://www.redalyc.org/articulo.oa?id=46142596016

Devis, J. (2000). Actividad física, deporte y salud. Madrid, España: Editorial Inde.

Ferrando, M. (2006). Posmodernidad y deporte. Madrid, España: Editorial Centro de Investigaciones Sociológicas - CIS. 
Fernández, P. (2010). Motivación hacia la práctica físico-deportiva. Valladolid, España: Editorial Paidotribo.

Moreno, J., Martínez, A., \& Torres, J. (2012). Motivaciones para la práctica fisco-deportiva en adolescentes españoles. España: Editorial Paidotribo.

Riffo, R. (2019). Gestión administrativa y de calidad en los centros escolares de los Chorrillos. Revista Scientific, 4(Ed. Esp.), 153-172, e-ISSN: 2542-2987. Recuperado de:

https://doi.org/10.29394/Scientific.issn.2542-2987.2019.4.E.9.153-172 


\section{Olger Estuardo Almache Tello}

e-mail: estuardo.almache@unae.edu.ec

Nacido en Suscal, provincia del Cañar, Ecuador, el 1 de

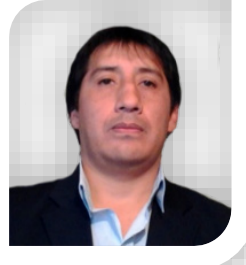
noviembre del año 1971. Máster en Cultura Física por la Universidad de Guayaquil (UG); Catedrático de deportes de la Universidad Nacional de Educación (UNAE); Catedrático de Cultura Física de la Universidad Católica de Cuenca (UCACUE), sede Azogues; Director del Departamento Técnico Metodológico de la Federación Deportiva del Cañar, desde el año 2001 hasta el año 2014; Docente de Cultura Física de la Escuela Gabriela Mistral, desde el año 1998 hasta el año 2011; Docente de Educación Física en el Centro Educativo la Esperanza, desde el año 1997 hasta el año 2001.

El contenido de este manuscrito se difunde bajo una Licencia de Creative Commons ReconocimientoNoComercial-Compartirlgual 4.0 Internacional 